\title{
Terapia Nutricional Enteral em Unidade de Terapia Intensiva: Infusão Versus Necessidades*
}

\section{Nutrition Support in an Intensive Care Unit: Delivery Versus Requirements}

\author{
Ana Carolina de Castro Teixeira ${ }^{1}$, Lúcia Caruso², Francisco G Soriano ${ }^{3}$
}

\section{RESUMO}

JUSTIFICATIVA E OBJETIVOS: Os pacientes internados em Unidade de Terapia Intensiva (UTI), freqüentemente apresentam depleção nutricional, tornando fundamental a monitoração diária da oferta nutricional. O objetivo desse estudo foi avaliar a adequação da Terapia Nutricional Enteral (TNE) na UTI adulto e identificar as causas de interrupção da administração enteral prescrita.

MÉTODO: Foi realizado um estudo observacional prospectivo de pacientes admitidos na UTI recebendo TNE exclusiva, durante 53 dias e infusão da dieta por meio de sistema fechado, infusão contínua (22 horas/dia) e sonda na posição pós-pilórica. A velocidade de administração da dieta iniciada com $25 \mathrm{~mL} / \mathrm{h}$ e progressão até atingir a meta nutricional conforme protocolo préestabelecido.

RESULTADOS: Foram acompanhados 33 pacientes com idade entre 18 e 85 anos, $58 \%$ do sexo masculino. Os principais diagnósticos de internação foram causas cardiovasculares $(27 \%)$ e choque séptico

1. Nutricionista, Ex-Aprimoranda do Serviço de Nutrição e Dietética do Hospital Universitário da USP.

2. Nutricionista do Serviço de Nutrição e Dietética do Hospital Universitário da USP.

3. Médico Chefe da Unidade da Terapia Intensiva Adulto do Hospital Universitário da USP.

*Recebido do Serviço de Nutrição e Dietética do Hospital Universitário da Universidade de São Paulo (USP), São Paulo, SP.

Apresentado em 06 de setembro de 2006

Aceito para publicação em 18 de outubro de 2006

Endereço para correspondência:

Ana Carolina de Castro Teixeira - *Serviço de Nutrição e Dietética/ HU-USP*

Av. Professor Lineu Prestes, 2565

Cidade Universitária $-1^{\circ} \mathrm{A}$

05508-900 São Paulo, SP

Fone/Fax: (11 ) 3039-9357

E-mail: snd@hu.usp.br

(C)Associação de Medicina Intensiva Brasileira, 2006
(21\%). O início da TNE ocorreu em 25,3 \pm 20 h após a admissão e atingiu a velocidade de meta nutricional em $32 \pm 20,1$ h. O volume diário prescrito, estabelecido a partir das necessidades previa atingir 26,1 $\pm 3,7 \mathrm{kcal} / \mathrm{kg}$ e $1,04 \mathrm{~g}$ de proteína/kg de peso corporal $\pm 0,1 \mathrm{~g} / \mathrm{kg}$. O volume administrado atingiu 19,5 $\pm 5,6 \mathrm{kcal} / \mathrm{kg}$ e $0,8 \mathrm{~g}$ de proteína $/ \mathrm{kg} \pm 0,2 \mathrm{~g} / \mathrm{kg}$, correspondendo a adequação de $74 \%$. Entre as causas da interrupção na administração da fórmula enteral, a maioria $(40,6 \%)$ foram para procedimentos de rotina relacionado ao paciente.

CONCLUSÕES: Os valores calóricos e protéicos atingidos com TNE neste serviço estão adequados conforme dados da literatura. Estes pacientes apresentaram grave instabilidade clínica interferindo negativamente na tolerância à nutrição enteral. Pode-se constatar que a contínua sistematização de rotinas e treinamento da equipe contribuiu positivamente em atingir os objetivos da terapia nutricional.

Unitermos: avaliação nutricional, nutrição enteral, terapia nutricional

\section{SUMMARY}

BACKGROUND AND OBJECTIVES: In critically ill patients nutritional deficiency is common. Considering this fact, the diary monitoring of energy delivered is extremely important. The objective of this study is to assess the adequacy of enteral nutrition (EN) in an Intensive Care Unit (ICU) and identify the reasons for interruptions in feeding.

METHODS: Prospective study of adult patients admitted to ICU in period of 53 days. Patients receiving continuous enteral tube feeding ( 22 hours/day) exclusively and post-pyloric tube feeding position were followed. The feeding volume started at $25 \mathrm{~mL} / \mathrm{h}$ and was increased until nutritional goal, guided by a feeding protocol. RESULTS: 33 patients between 18 and 85 years old were studied. $58 \%$ were male. The main admission diagnoses were cardiovascular diseases $(27 \%)$ and 
septic shock (21\%). The mean time to feeding was 25.3 hours after the patient admission and nutritional goal was achieved in a mean time of 32 hours. The total volume prescribed per day was sufficient to guarantee a mean of $26.1 \mathrm{kcal} / \mathrm{kg}$ of body weight and $1.04 \mathrm{~g}$ of protein $/ \mathrm{kg}$ of body weight. Patients received a mean of $19.5 \mathrm{kcal} / \mathrm{kg}$ of body weight and $0.8 \mathrm{~g}$ of protein/kg of body weight, which correspond a $74 \%$ of adequacy. Interruptions of feeding for routine procedures related to patients accounted for $40.6 \%$ of the total reasons.

CONCLUSIONS: The nutritional support is adequate considering the literature for these patients, who clinical instability causes gastrointestinal intolerance. The effective participation of the Nutrition Therapy Team can contribute positively on nutritional therapy.

Key Words: enteral nutrition, nutrition assessment, nutrition therapy

\section{INTRODUÇÃO}

Em pacientes internados em unidades de terapia intensiva, a depleção nutricional é freqüente, já que a resposta metabólica ao estresse, conhecida como resposta de fase aguda, promove intenso catabolismo e mobilização de proteínas para reparo de tecidos lesados e fornecimento de energia.

A reação de fase aguda, seguida de imobilidade prolongada e a dificuldade de alimentação potencializam o catabolismo e predispõem ao déficit nutricional. Dessa forma, o paciente em reação de fase aguda como conseqüência do hipermetabolismo está em risco nutricional, independentemente do estado nutricional prévio. A situação clínica expõe ao risco nutricional pelas alterações que promove no metabolismo. Além disso, fatores como idade avançada, condição sócio-econômica e desnutrição preexistente podem intensificar a agressão ao estado nutricional' .

Quando há depleção nutricional, a resposta imunológica é deprimida, o processo de cicatrização é comprometido, ocorrem alterações na composição corporal e na função dos órgãos, além de outras conseqüências que levam à maior probabilidade de ocorrência de infecções, escaras, entre outras complicações. Isso resulta em incremento na morbidade e na mortalidade, além de levar ao prolongamento do tempo de internação, bem como elevar o número de re-internações, fatores esses associados com maior custo de assistência ${ }^{2-4}$.

Considerando que a terapia nutricional precoce e ade- quada no paciente grave é um importante fator na promoção da saúde, diminuição do estresse fisiológico e manutenção da imunidade, avaliar a eficácia desse tratamento é fundamental ${ }^{5}$.

Apesar da importância da adequada ingestão de nutrientes e energia, os pacientes internados em UTI, freqüentemente recebem um valor energético inferior as suas necessidade. Os fatores que impedem o adequado aporte nutricional enteral incluem os relacionados à intolerância da dieta (vômitos, diarréia, resíduo gástrico, distensão abdominal, etc.), os associados às práticas de rotina de enfermagem (manipulação do paciente, administração de medicamentos, etc.) e outras rotinas (procedimentos, exames) $)^{5-8}$. A atenção nutricional e o conhecimento dos fatores que impedem a efetiva administração da TNE permitem a adoção de medidas visando o aporte calórico-protéico adequado aos pacientes graves.

Este estudo teve por objetivo avaliar a adequação da Terapia Nutricional Enteral (TNE) de pacientes internados na Unidade de Terapia Intensiva (UTI) adulto que recebem nutrição exclusivamente por via enteral e identificar as causas de interrupção da administração da fórmula enteral prescrita.

\section{MÉTODO}

Estudo de caráter observacional e prospectivo, com participação de pacientes de ambos os sexos, com idade entre 18 e 85 anos, admitidos na UTI. O projeto foi aprovado pelo Comitê de Ética em Pesquisa da Instituição (CEP 603/05) e o paciente só entrou no estudo após assinar o termo de consentimento (Anexo 1).

Foram incluídos no estudo pacientes com terapia nutricional exclusivamente por via enteral e que a receberam por, pelo menos, 72 horas. Foram excluídos pacientes com contra-indicação de TNE ou que receberam dieta parenteral e/ou oral concomitante.

A coleta de dados iniciou-se no primeiro dia de introdução da nutrição enteral e o acompanhamento dos pacientes foi realizado até o momento de descontinuação da terapia nutricional, óbito, ou alta da unidade.

Todos os pacientes com indicação de TNE foram avaliados pelo nutricionista, para estimativa das necessidades de energia e de proteínas, de acordo com parâmetros estabelecidos para cada diagnóstico, conforme mostra a tabela 1. 
Tabela 1 - Fórmulas para Estimativa de Necessidades Diárias de Energia e Proteínas.

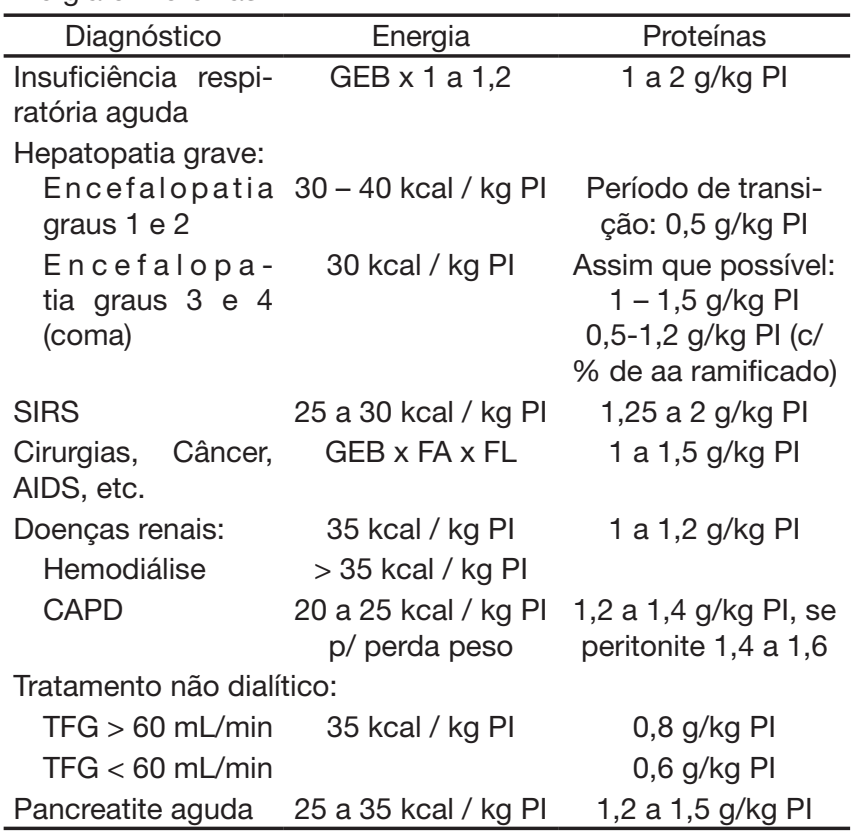

Fonte: adaptado de Cerra e col. $1997^{13}$; Klein e col. ${ }^{14}$; Espen, $2000{ }^{15}$, Aspen, $2002{ }^{16}$.

GEB = gasto energético basal (calculado a partir da fórmula proposta por Harris e Benedict); FA: fator atividade; FL = fator lesão; $\mathrm{PI}=$ peso ideal; aa: aminoácidos; SIRS = síndrome da resposta inflamatória sistêmica; AIDS = síndrome da imunodeficiência adquirida; CAPD = diálise peritoneal; TFG = taxa de filtração glomerular.

O peso corporal utilizado para o cálculo das necessidades de energia e proteína foi estimado a partir de tabelas padrão de referência (peso ideal) ${ }^{9-12}$.

A partir da estimativa determinou-se o tipo de fórmula enteral e a velocidade de infusão da dieta para atingir a meta nutricional.

Os pacientes receberam nutrição enteral (NE) por meio de sonda locada na posição pós-pilórica (após confirmação por radiografia) e fórmulas enterais poliméricas em 3 opções de formulação ( $1 \mathrm{kcal} / \mathrm{mL}$ sem fibras; 1,5 $\mathrm{kcal} / \mathrm{mL}$ sem fibras e $1 \mathrm{kcal} / \mathrm{mL}$ com fibras).

Todas as dietas foram administradas em sistema fechado através de infusão contínua por bomba infusora, com previsão de $22 \mathrm{~h} / \mathrm{dia}$, (considerou-se 2 horas de pausa para rotina de enfermagem e administração de medicamentos). A velocidade de infusão inicial foi de 25 $\mathrm{mL} / \mathrm{h}$ e evoluiu para $10 \mathrm{~mL} / \mathrm{h}$ a cada 4 horas, de acordo com a tolerância do paciente, seguindo o protocolo préestabelecido ${ }^{17}$. Esse foi desenvolvido em conjunto pelas equipes médica, de enfermagem e de nutrição, para a introdução e evolução da velocidade de administração da fórmula enteral em sistema contínuo.

Considerou-se como diarréia episódios de três ou mais evacuações líquidas ou semilíquidas em 24 horas $^{18}$.
Os dados foram coletados pelas próprias pesquisadoras, nos prontuários médicos, nas anotações diárias de equipe de enfermagem em ficha específica.

Os resultados foram compilados no programa Microsoft ${ }^{\circledR}$ Excel.

O valor nutricional (valor calórico e proteínas) da fórmula enteral prescrita (volume planejado segundo prescrição diária de acordo com as necessidades calculadas) e da administrada (volume total diário realmente infundido), foi calculado de duas maneiras:

- Total diário de energia (kcal) e proteína (g) de cada paciente e média;

- Total diário expresso em relação ao peso corporal: energia $(\mathrm{kcal} / \mathrm{kg})$ e proteína $(\mathrm{g} / \mathrm{kg})$ de cada paciente e respectiva média;

A adequação foi obtida comparando-se esses valores a partir das seguintes fórmulas:

1. adequação do volume prescrito (\%) = volume prescrito $\times 100$

volume calculado

2. adequação do volume infundido (\%) = volume infundido $\times 100$

volume prescrito

Foi considerado como adequado o volume infundido superior a $90 \%$ do volume diário total planejado.

\section{RESULTADOS}

O estudo foi realizado por período de 53 dias, onde foram acompanhados 33 pacientes, com idade média de 57 anos (variando entre 18 e 85 anos) e $58 \%$ do sexo masculino.

O principal diagnóstico de internação foi o de causas cardiovasculares (27\%), e em seguida, as internações por choque séptico/sepse (21\%) (Figura 1).

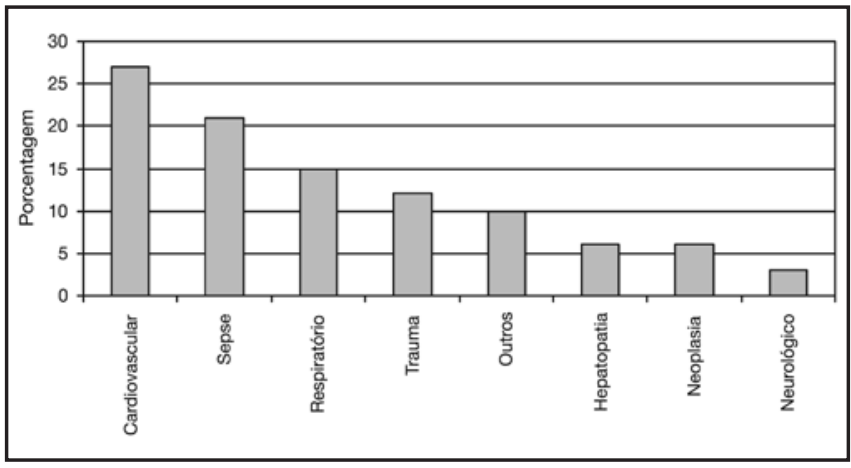

Figura 1 - Distribuição dos Pacientes segundo o Diagnóstico de Internação.

Fonte: Hospital Universitário - USP, 2005. 
A nutrição enteral (NE) é considerada o método de escolha para pacientes em UTI que necessitam de Terapia Nutricional. O início da TNE neste estudo foi em média $25,3 \pm 20$ horas, em relação à sua admissão. Esse dado é bastante satisfatório, uma vez que a introdução precoce da dieta (entre 24 e 48 horas após admissão na unidade) traz benefícios ao paciente, prevenindo o desgaste nutricional e conseqüentemente a desnutrição ${ }^{19,20}$.

A média de permanência na UTI foi de $15 \pm 10$,8 dias e em TNE de 12,8 $\pm 11,2$ dias. O GET (Gasto Energético Total) médio calculado foi de 1694,8 $\pm 253,4 \mathrm{kcal}(25,8$ $\pm 3,5 \mathrm{kcal} / \mathrm{kg})$ e $72,8 \pm 13,1 \mathrm{~g}$ de proteína $(1,1 \pm 0,1 \mathrm{~g} /$ $\mathrm{kg}$ ), o que é esperado, uma vez que as maiores causas de internação são por distúrbios cardiovasculares em que a recomendação é de uma dieta normocalórica (25 a $30 \mathrm{kcal} / \mathrm{kg}$ ) e normoprotéica (entre 0,8 e $1 \mathrm{~g} / \mathrm{kg}$ ) e nos casos de sepse, onde a dieta deve ser normocalórica (25 a $30 \mathrm{kcal} / \mathrm{kg}$ ), porém hiperprotéica (1,25 a $2 \mathrm{~g} / \mathrm{kg})$. Os resultados mostraram que a quantidade diária de calorias e proteínas prescritas foram, respectivamente, de $1715,7 \pm 250,8 \mathrm{kcal}(26,1 \pm 3,7 \mathrm{kcal} / \mathrm{kg})$ e $68,6 \pm 10,1 \mathrm{~g}$ $(1,04 \pm 0,1 \mathrm{~g} / \mathrm{kg})$. Esses valores estão bem próximos da média das necessidades calculadas, o que indica uma adequação entre o prescrito e as necessidades do paciente (Tabela 2). Esses resultados estão de acordo com o encontrado na literatura. Adam e Batson ${ }^{21}$ que analisaram cinco UTI da Inglaterra, obtiveram a média de necessidades de energia prescrita variando entre $76 \%$ e $100 \%$.

Tabela 2 - Média de Valores de Energia e Proteína Calculado e Prescrito

\begin{tabular}{lccc}
\hline & Calculado & Prescrito & Adequação \\
\hline $\begin{array}{l}\text { Valor ca- } \\
\text { lórico }\end{array}$ & $25,8 \pm 3,5 \mathrm{kcal} / \mathrm{kg}$ & $26,1 \pm 3,7 \mathrm{kcal} / \mathrm{kg}$ & $101 \%$ \\
Proteína & $1,1 \pm 0,1 \mathrm{~g} / \mathrm{kg}$ & $1,04 \pm 0,1 \mathrm{~g} / \mathrm{kg}$ & $94 \%$ \\
\hline
\end{tabular}

Fonte: Hospital Universitário - USP, 2005.

Nem sempre os valores calculados são passíveis de serem atingidos na prescrição diária devido as fórmulas enterais disponíveis no mercado. Isto ocorre principalmente em relação as proteínas, onde foi constatada uma adequação de 94\%. A maioria das fórmulas de sistema fechado é normocalórica e normoprotéica ou então hipercalórica e hiperprotéica, por isso é difícil atingir as necessidades de proteínas sem ultrapassar as necessidades de calorias. Para o adequado atendimento dessas necessidades nessa população é interessante a utilização de fórmulas normocalóricas e hiperprotéicas, que atualmente estão disponíveis por alguns fabricantes.
A administração da NE é prejudicada pelos fatores que impedem a sua infusão contínua. Neste trabalho, o aporte calórico médio administrado foi de 1280,16 $\pm 368,2$ $\mathrm{kcal}(19,5 \pm 5,6 \mathrm{kcal} / \mathrm{kg})$ e de proteínas $50,8 \pm 14,7 \mathrm{~g}(0,8$ $\pm 0,2 \mathrm{~g} / \mathrm{kg}$ ) o que representa, respectivamente uma adequação de $74,4 \%$ e $74,1 \%$ em relação ao prescrito (Tabela 3). De Jonghe e col. ${ }^{7}$ também obtiveram resultados semelhantes, onde a média do GET prescrito foi de $78 \%$ e o realmente administrado foi de $71 \%$.

Tabela 3 - Média de Valores de Energia e Proteína Prescrito, Administrado e Adequação.

\begin{tabular}{lccc}
\hline \multicolumn{1}{c}{ Prescrito } & Administrado & Adequação \\
\hline $\begin{array}{l}\text { Valor ca- } \\
\text { lórico }\end{array}$ & $26,1 \pm 3,7 \mathrm{kcal} / \mathrm{kg}$ & $19,5 \pm 5,6 \mathrm{kcal} / \mathrm{kg}$ & $74,40 \%$ \\
Proteína & $1,04 \pm 0,1 \mathrm{~g} / \mathrm{kg}$ & $0,8 \pm 0,2 \mathrm{~g} / \mathrm{kg}$ & $74,10 \%$ \\
\hline
\end{tabular}

Fonte: Hospital Universitário - USP, 2005.

Já McClave e col. ${ }^{22}$, demonstraram uma taxa de administração média de $51,6 \%$, mas somente $14 \%$ dos pacientes atingiram o objetivo calórico após 72 horas do início da NE.

Estudos mostram que a porcentagem de administração da NE em UTI é variável e pode atingir de $50 \%$ a $100 \%$ do objetivo calórico desejado ${ }^{7,21-24}$. As figuras 2 e 3 apresentam a diferença entre o administrado e o prescrito de cada um dos 33 pacientes acompanhados. Pode-se observar que apesar da média ter atingido valores de adequação muito bons, alguns pacientes tiveram uma administração inadequada. Isto se deve a fatores que não permitiram a contínua infusão como previsto.

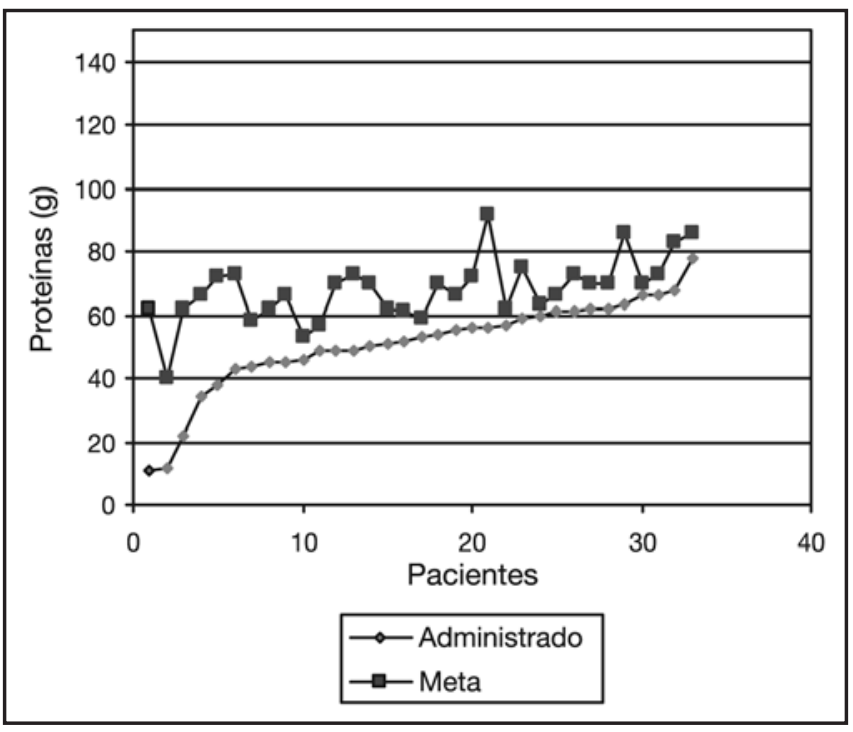

Figura 2 - Distribuição de Proteínas Administradas e Prescritas segundo os Pacientes.

Fonte: Hospital Universitário - USP, 2005. 


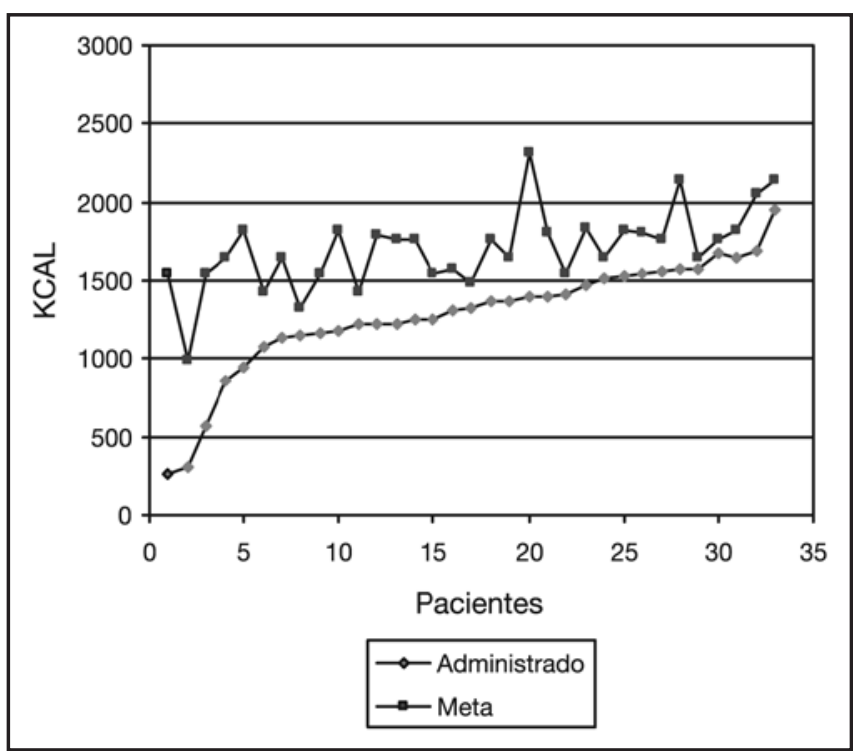

Figura 3 - Distribuição do Valor Calórico (kcal) Administrado e Prescrito segundo os Pacientes.

Fonte: Hospital Universitário - USP, 2005.

A administração de NE é dificultada por fatores diretamente relacionados com a Terapia Intensiva (instabilidade hemodinâmica, jejum para procedimentos e exames, dentre outros). Ocorrem também problemas mecânicos com a sonda nasoenteral, como obstrução, mau posicionamento, demora para a sua repassagem. Os principais fatores que impediram a adequada administração da NE foram as interrupções de rotina relacionadas ao paciente $(40,6 \%)$, como por exemplo, pausas para administração de medicamentos por sonda, pausas para banho, etc. Não foi possível quantificar essas pausas já que não existem registros específicos para anotação em fichas de controle. Uma observação pertinente é de que os dados foram coletados logo após a implantação do sistema fechado e do protocolo de infusão contínua. Este fato pode ter interferido na falta

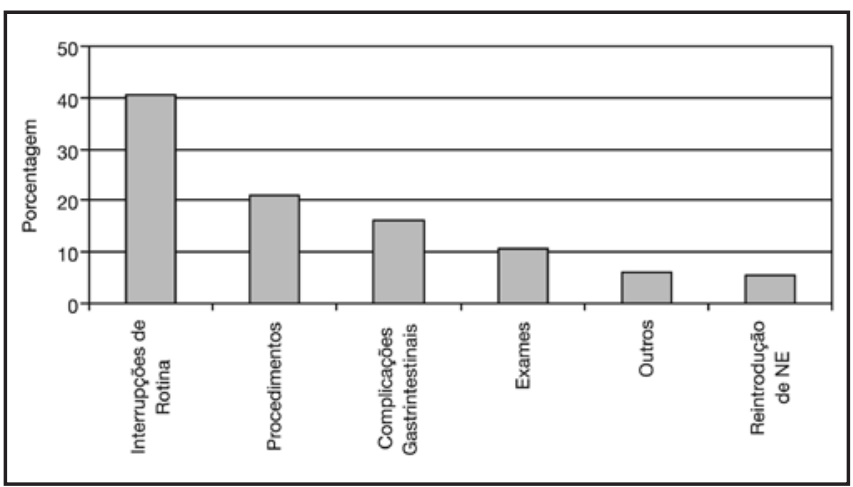

Figura 4 - Distribuição das Causas de não Recebimento da Nutrição Enteral.

Fonte: Hospital Universitário - USP, 2005. de especificação detalhada das pausas.

A segunda principal causa de interrupção da dieta foi jejum para procedimentos (extubação, traqueostomia e repassagem da sonda) em $21 \%$ dos casos (Figura 4).

Em um estudo realizado por Elpern e col. ${ }^{5}$, com 39 pacientes de UTI recebendo NE em infusão contínua, a interrupção da administração da fórmula enteral por exames e procedimentos foi o principal motivo com $35,7 \%$ das ocorrências.

No presente estudo o refluxo representou 12,4\% (Figura 5) das causas de interrupção da NE. Tal resultado é inferior ao encontrado na literatura. No estudo realizado por Elpern e col. ${ }^{5}$ as causas relacionadas à interrupção da dieta devido ao refluxo foram de $28 \%$ e Petros e Engelmann ${ }^{25}$ encontraram $31,9 \%$ de incidência de refluxo como causa de interrupção da dieta.

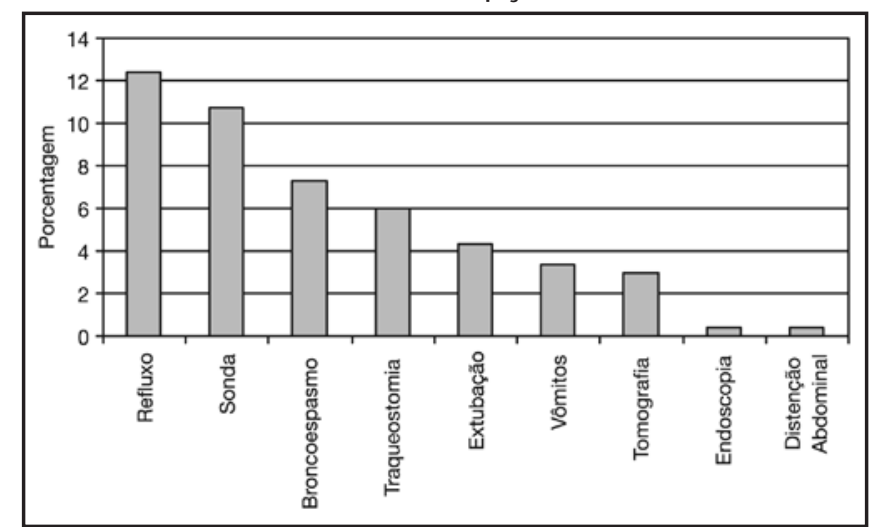

Figura 5 - Distribuição das Causas de não Recebimento de Nutrição Enteral segundo os Tipos de Exames, Procedimentos e Complicações Gastrintestinais.

Fonte: Hospital Universitário - USP, 2005.

Uma provável explicação para essa menor incidência de refluxo, se comparado com o encontrado na literatura, é a locação da sonda na posição pós-pilórica, permitindo melhor tolerância da dieta. Sabe-se que pacientes críticos podem apresentar gastroparesia e aumento do resíduo gástrico, sendo a estase gástrica uma das principais causas mensuráveis que impedem a administração da NE. Um ponto a ser considerado é que na literatura, estudos comparando posicionamento da sonda pré e pós-pilórica mostram-se um tanto inconclusivos ${ }^{26-28}$.

Estudos indicam que a incidência de diarréia em pacientes com NE varia entre $2 \%$ e $63 \%{ }^{29-31}$ dependendo do parâmetro utilizado para a classificação de diarréia. No presente estudo, $36 \%(n=12)$ dos pacientes apresentaram pelo menos um episódio de diarréia. Em estudo realizado por Elpern e col. ${ }^{5}$ constataram-se diarréia em $72 \%$ dos pacientes, enquanto que Petros e Engelmann ${ }^{25}$, obtiveram apenas $9,6 \%$ de incidência de diarréia. Isso 
demonstra a grande variabilidade de dados encontrados quando se refere à incidência de diarréia.

Cabe salientar que a diarréia não foi causa de interrupção da dieta no presente estudo.

\section{CONCLUSÃO}

A população predominante no estudo foi de homens cujo principal diagnóstico de internação foi relacionado às disfunções cardiovasculares e choque séptico. Apesar da média de idade ser de 57 anos, parcela considerável, $48 \%$ era composta de idosos (> 65 anos). O volume prescrito de fórmula enteral atingiu as necessidades nutricionais de energia e esteve bem próximo de atingir as necessidades de proteína. No entanto, a adequação do volume administrado em relação ao volume prescrito não alcançou o total planejado. Nesse sentido é importante considerar que na literatura, os estudos com pacientes críticos apresentam valores semelhantes aos encontrados no presente trabalho.

As intercorrências relacionadas à tolerância gastrintestinal do paciente foram menores se comparadas às demais causas de interrupção da NE. Provavelmente esse resultado é conseqüência da utilização do protocolo para evolução da TNE em infusão contínua. Além disso, cabe enfatizar o grande envolvimento das equipes em seguir o protocolo. Outro fator que provavelmente contribuiu para melhor evolução da NE foi a sonda na posição pós-pilórica, que se mostrou favorável à prevenção de resíduo gástrico e intercorrências relacionadas.

Os outros fatores que interferiram na administração da NE podem ser minimizados. O tempo de jejum para os exames e procedimentos pode ser reduzido se for realizado um planejamento adequado para a interrupção e re-introdução da infusão da dieta. As pausas para procedimentos de enfermagem e fisioterapia também podem ser diminuídas se forem realizadas de formas sistematizada e articulada. O continuo treinamento da equipe de enfermagem pode diminuir as intercorrências relacionadas à obstrução e perda da sonda enteral, assim como uma rápida troca da sonda nesses casos.

Dessa forma, a presença de Equipe Multiprofissional de Terapia Nutricional (EMTN), o seguimento do protocolo para infusão da dieta e o constante treinamento e sistematização do atendimento contribuem para meIhorar a administração da TNE em Terapia Intensiva, fundamental para a assistência ao paciente grave em risco nutricional.

\section{Anexo 1}

\section{TERMO DE CONSENTIMENTO LIVRE E ESCLARECIDO}

Você está sendo convidado (a) a participar de um estudo, que tem por objetivo avaliar a adequação da terapia nutricional por meio de sondas para alimentação: "Terapia nutricional via enteral na Unidade de Terapia Intensiva: monitorização infusão x necessidades".

Este estudo será realizado por nutricionistas, que calcularão as necessidades nutricionais utilizando medidas de peso e altura estimadas, e levantará alguns dados a partir de informações referentes a administração da nutrição por sonda contidas no prontuário do paciente.

O paciente não sofrerá nenhum prejuízo nutricional devido ao nosso levantamento.

A participação neste estudo é voluntária e a identidade do paciente será mantida em sigilo. Sendo assim, você pode se recusar a participar, ou interromper a participação, sem nenhum prejuízo para o atendimento que o paciente recebe nesta Instituição.

A sua colaboração neste estudo ajudará este hospital a melhorar cada vez mais a qualidade do atendimento ao paciente da Unidade de Terapia Intensiva.

"Declaro que li e entendi as informações que me foram transmitidas e concordo em participar do estudo, ou permitir que o paciente pelo qual sou responsável participe "

Nome do paciente Número de atendimento

$\overline{\text { Nome do Responsável legal Número do documento de identidade }}$

Grau de parentesco Assinatura do Responsável legal

\section{I}

Pesquisador Responsável:

Eu, , responsável pela pesquisa "Terapia nutricional via enteral na Unidade de Terapia Intensiva: monitorização infusão x necessidades" declaro que obtive espontaneamente o consentimento para realizar este estudo.

Ass: São Paulo,

Contatos: Lúcia Caruso - nutricionista do SND- HU - USP: fone: 30399357

Ana Carolina de Castro Teixeira - aprimoranda do SND - HU - USP: fone: 3039-9357 Comitê de Ética em Pesquisa (CEP):

Endereço: Av. Professor Lineu Prestes, 2565 Cidade Universitária - São Paulo - SP

05508-900 - FONE: 30399457 - FAX: 30399452 e-mail: cep@hu.usp.br 


\section{REFERÊNCIAS}

01. Lavery GG, Glover P - The metabolic and nutritional response to critical illness. Curr Opin Crit Care, 2000;6:233-238.

02. Heyland DK, Dhaliwal R, Drover JW et al - Canadian clinical practice guidelines for nutrition support in mechanically ventilated, critically ill adult patients. JPEN J Parenter Enteral Nutr, 2003;27:355-373.

03. Waitzberg DL, Correia MI - Nutritional assessment in the hospitalized patient. Curr Opin Clin Nutr Metab Care, 2003;6:531-538.

04. Correia MI, Waitzberg DL - The impact of malnutrition on morbidity, mortality, length of hospital stay and costs evaluated through a multivariate model analysis. Clin Nutr, 2003;22:235-239.

05. Elpern EH, Stutz L, Peterson S et al - Outcomes associated with enteral tube feedings in a medical intensive Care Unit. Am J Crit Care, 2004;13:221-227.

06. Garvin CG, Brown RO - Nutritional support in the intensive care unit: are patients receiving what is prescribed? Crit Care Med, 2001;29:204-205.

07. De Jonghe B, Appere-de-Vechi C, Fournier M et al - A prospective survey of nutritional support practices in intensive care unit patients: what is prescribed? What is delivered? Crit Care Med, 2001;29:8-12.

08. O'Leary-Kelley CM, Puntillo KA, Barr $\mathrm{J}$ et al - Nutritional adequacy in patients receiving mechanical ventilation who are fed enterally. Am J Crit Care, 2005;14:222-231.

09. Burr ML, Phillips KM - Anthropometric norms in the elderly. Br J Nutr, 1984;51:165-169.

10. Glynn CC, Greene GW, Winkler MF et al - Predictive versus measured energy expenditure using limits-of-agreement analysis in hospitalized, obese patients. JPEN J Parenter Enteral Nutr, 1999;23:147-154

11. Krause MV, Mahan LK - Alimentação, Nutrição e Dietoterapia. São Paulo: Roca, 10 Ed, São Paulo, Roca, 2002.

12. Cutts ME, Dowdy RP, Ellersieck MR et al - Predicting energy needs in ventilator-dependent critically ill patients: effect of adjusting weight for edema or adiposity. Am J Clin Nutr, 1997;66:1250-1256.

13. Cerra FB, Benitez MR, Blackburn GL et al - Applied nutrition in ICU patients. A consensus statement of the American College of Chest Physicians. Chest, 1997;111:769-778.

14. Klein CJ, Stanek GS, Wiles CE $3^{\text {rd }}$ - Overfeeding macronutrients to critically ill adults: metabolic complications. J Am Diet Assoc, 1998;98:795-806.

15. European Society of Parenteral and Enteral Nutrition (ESPEN), Sobotka L - Basics in Clinical Nutrition. Prague, Galén, 2000.

16. ASPEN Board of Directors and the Clinical Gluidelines Task Force. Guidelines for the use of parenteral and enteral nutrition in adult and pediatric patients. JPEN J Parenter Enteral Nutr, 2002;26:(Suppl1):1SA138SA.

17. Caruso L, Teixeira ACC, Maia FOM et al - Elaboração de protocolo em terapia nutricional enteral: relato de experiência. In: XII Congresso Brasileiro de Medicina Intensiva - Adulto, Pediátiro - Neonatal, 2006, Recife. RBTI, 2006;285.

18. Mahan K, Arlin MT - Doenças Intestinais, em: Mahan K, Arlin MT - Krause: Alimentos, Nutrição \& Dietoterapia, 9ª Ed, São Paulo, Roca, 1998.

19. Barr J, Hecht M, Flavin KE et al - Outcomes in critically ill patients before and after the implementation of an evidence-based nutritional management protocol. Chest, 2004;125:1446-1457.

20. Heyland DK, Dhaliwal R, Day A et al - Validation of the Canadian clinica practice guidelines for nutrition support in mechanically ventilated, critically ill adult patients: results of a prospective observational study. Crit Care Med, 2004;32:2260-2266.

21. Adam S, Batson S - A study of problems associated with the delivery of enteral feed in critically ill patients in five ICUs in the UK. Intensive Care Med, 1997;23:261-266.

22. McClave SA, Sexton LK, Spain DA et al - Enteral tube feeding in the intensive care unit: factors impending adequate delivery. Crit Care Med, 1999;27:1252-1256.

23. Heyland D, Cook DJ, Winder B et al - Enteral nutrition in the critically ill patient: a prospective survey. Crit Care Med, 1995;23:1055-1060.

24. Webster NR, Galley HF - Nutrition in the critically ill patient. J R Coll Surg Edinb, 2000;45:373-379.

25. Petros S, Engelmann L - Enteral nutrition delivery and energy expenditure in medical intensive care patients. Clin Nutr, 2006;25:51-59.

26. Williams TA, Leslie GD - A review of the nursing care of enteral feeding tubes in critically ill adults: part I. Intensive Crit Care Nurs, 2004;20:330343.

27. Jacobs DG, Jacobs DO, Kudsk KA et al - Practice management for nutritional support of the trauma patient. J Trauma, 2004;57:660-679.

28. Marik PE, Zaloga GP - Gastric versus post-pyloric feeding: a systematic review. Crit Care, 2003;7:R46-R51.

29. D'Souza AL, Rajkumar C, Cooke $\mathrm{J}$ et al - Probiotics in prevention of antibiotic associated diarrhoea: meta-analysis. BMJ, 2002;324:1361-1366.

30. Cremonini F, DI Caro S, Nista EC et al - Meta-analysis: the effect of probiotic administration on antibiotic associated diarrhoea. Aliment Pharmacol Ther, 2002;16:1461-1467.

31. Bleichner G, Blehaut $\mathrm{H}$, Mentec $\mathrm{H}$ et al - Saccharomyces boulardii prevents diarrhea in critically ill tube-fed patients. A multicenter, randomized, double-blind placebo-controlled trial. Intensive Care Med, 1997;23:517523. 